\title{
Title: Shapes and Vorticities of Superfluid Helium Nanodroplets
}

\section{Authors:}

Luis F. Gomez, ${ }^{1}$ Ken R. Ferguson, ${ }^{2}$ James P. Cryan, ${ }^{3}$ Camila Bacellar, ${ }^{3,4}$ Rico Mayro P. Tanyag, ${ }^{1}$ Curtis Jones, ${ }^{1}$ Sebastian Schorb, ${ }^{2}$ Denis Anielski, ${ }^{5,6}$ Ali Belkacem, ${ }^{3}$ Charles Bernando, ${ }^{7}$ Rebecca Boll, ${ }^{5,6,8}$ John Bozek, ${ }^{2}$ Sebastian Carron, ${ }^{2}$ Gang Chen, ${ }^{9} \S$ Tjark Delmas, ${ }^{10}$ Lars Englert, ${ }^{11}$ Sascha W. Epp, ${ }^{5,6}$ Benjamin Erk, ${ }^{5,6,8}$ Lutz Foucar, ${ }^{6,12}$ Robert Hartmann, ${ }^{13}$ Alexander Hexemer, ${ }^{9}$ Martin Huth, ${ }^{13}$ Justin Kwok, ${ }^{14}$ Stephen R. Leone, ${ }^{3,4,15}$ Jonathan H. S. Ma, ${ }^{3,16}$ Filipe R. N. C. Maia, ${ }^{17}$ Erik Malmerberg, ${ }^{18}$ Stefano Marchesini, ${ }^{9}$ Daniel M. Neumark, ${ }^{3,4}$ Billy Poon, ${ }^{18}$ James Prell, ${ }^{4}$ Daniel Rolles, ${ }^{6,8,12}$ Benedikt Rudek, ${ }^{5,6} \dagger$ Artem Rudenko, ${ }^{5,6,19}$ Martin Seifrid, ${ }^{1}$ Katrin R. Siefermann, ${ }^{3}+\dagger$ Felix P. Sturm, ${ }^{3}$ Michele Swiggers, ${ }^{2}$ Joachim Ullrich,${ }^{5,6} \dagger$ Fabian Weise, ${ }^{3} \S \S$ Petrus Zwart, ${ }^{18}$ Christoph Bostedt, ${ }^{2,20 *}$ Oliver Gessner, ${ }^{3 *}$ Andrey F. Vilesov ${ }^{1,7 *}$

\section{Affiliations:}

${ }^{1}$ Department of Chemistry, University of Southern California, Los Angeles, California 90089, USA.

${ }^{2}$ Linac Coherent Light Source, LCLS, SLAC National Accelerator Laboratory, 2575 Sand Hill Road, Menlo Park, California 94025, USA.

${ }^{3}$ Ultrafast X-ray Science Laboratory, Chemical Sciences Division, Lawrence Berkeley National Laboratory, Berkeley, California 94720, USA.

${ }^{4}$ Department of Chemistry, University of California Berkeley, Berkeley, California 94720, USA.

${ }^{5}$ Max-Planck-Institut für Kernphysik, Saupfercheckweg 1, 69117 Heidelberg, Germany.

${ }^{6}$ Max Planck Advanced Study Group at the Center for Free-Electron Laser Science (CFEL), Notkestraße 85, 22607 Hamburg, Germany.

${ }^{7}$ Department of Physics and Astronomy, University of Southern California, Los Angeles, California 90089, USA.

${ }^{8}$ Deutsches Elektronen-Synchrotron (DESY), Notkestraße 85, 22607 Hamburg, Germany.

${ }^{9}$ Advanced Light Source, Lawrence Berkeley National Laboratory, Berkeley, California 94720, USA.

${ }^{10}$ Center for Free-Electron Laser Science (CFEL), Deutsches Elektronen-Synchrotron (DESY), Notkestraße 85, 22607 Hamburg, Germany.

${ }^{11}$ Max-Planck-Institut für extraterrestrische Physik, Giessenbachstraße, 85741 Garching, Germany.

${ }^{12}$ Max-Planck-Institut für Medizinische Forschung, Jahnstrasse 29, 69120 Heidelberg, Germany.

${ }^{13}$ PNSensor GmbH, Otto-Hahn-Ring 6, 81739 München, Germany.

${ }^{14}$ Mork Family Department of Chemical Engineering and Materials Science, University of Southern California, Los Angeles, California 90089, USA.

${ }^{15}$ Department of Physics, University of California Berkeley, Berkeley, California 94720, USA.

${ }^{16}$ Department of Physics, The Chinese University of Hong Kong, Hong Kong, China.

${ }^{17}$ NERSC, Lawrence Berkeley National Laboratory, Berkeley, California 94720, USA.

${ }^{18}$ Physical Biosciences Division, Lawrence Berkeley National Laboratory, Berkeley, California 94720, USA.

${ }^{19}$ J.R. MacDonald Laboratory, Department of Physics, Kansas State University, Manhattan, Kansas 66506, USA.

${ }^{20}$ PULSE Institute, Stanford University and SLAC National Accelerator Laboratory, 2575 Sand Hill Road, Menlo Park, California 94025, USA.

*To whom correspondence and requests for materials should be addressed. E-mail: bostedt@slac.stanford.edu (C.B.); ogessner@lbl.gov (O.G.); vilesov@usc.edu (A.F.V.)

§Present Address: Shanghai Synchrotron Radiation Facility, Shanghai Institute of Applied Physics, Chinese Academy of Sciences, Shanghai 201204, China.

tPresent Address: Department of Cell and Molecular Biology, Uppsala University, Husargatan 3, Uppsala 75124, Sweden. 
$†$ Present Address: Physikalisch-Technische Bundesanstalt, Bundesallee 100, D-38116 Braunschweig, Germany.

$\$$ Present Address: Leibniz Institute of Surface Modification (IOM), Permoserstraße 15, 04318 Leipzig, Germany.

$\S \S$ Present Address: Berliner Glas KGaA Herbert Kubatz GmbH \& Co., Waldkraiburger Str. 5, 12347 Berlin, Germany.

\begin{abstract}
:
Helium nanodroplets are considered ideal model systems to explore quantum hydrodynamics in self-contained, isolated superfluids. However, exploring the dynamic properties of individual droplets is experimentally challenging. Here, we investigate the rotation of single, isolated, superfluid ${ }^{4} \mathrm{He}$ droplets, containing $\sim 10^{8}-10^{11}$ atoms, using single-shot femtosecond X-ray coherent diffractive imaging. The formation of quantum vortex lattices inside the droplets is confirmed by observing characteristic Bragg patterns from Xe clusters trapped in the vortex cores. The vortex densities are up to five orders of magnitude larger than observed in bulk liquid He. The droplets exhibit large centrifugal deformations but retain axially symmetric shapes at angular velocities well beyond the stability range of viscous classical droplets.
\end{abstract}




\section{Main Text:}

The discoveries of superconductors, superfluids, and Bose-Einstein condensates (1-2) reveal that a large number of particles can occupy a single quantum state that extends across macroscopic length scales. A notable example is superfluid ${ }^{4} \mathrm{He}$ (3-6), which, below a critical temperature of $T_{\lambda}=2.17 \mathrm{~K}$, lacks any viscosity, and its motion is described by a single wavefunction (1-2, 5-7). Isolated $\mathrm{He}$ nanodroplets were employed to study the onset of superfluidity through the observation of frictionless, quantized rotation of embedded molecules surrounded with varying numbers of ${ }^{4} \mathrm{He}$ atoms $(8)$. However, the unambiguous demonstration of a quantum mechanical state of motion of an entire helium nanodroplet remains challenging.

In a finite droplet, any manifestation of liquid flow must involve rotational motion, which in a superfluid embodies itself in quantum vortices (6-7). Indeed, the formation of regular arrays of parallel vortices was detected in a rotating bucket filled with superfluid He (9-10). However, surprisingly little is known about quantum rotation in superfluid droplets. Calculations predict that vortices may exist in ${ }^{4} \mathrm{He}$ droplets as small as a few nanometers in diameter (11-13) but experimental studies of this elusive phenomenon remain challenging (14). Recently, traces of vortices were detected in He droplets about $1 \mu \mathrm{m}$ in diameter (15). These exploratory experiments, however, did not provide detailed hydrodynamic properties of the spinning droplets, such as their shapes or the spatial arrangements of the vortices they contain. Herein, we study the rotation of single, isolated superfluid He nanodroplets via coherent scattering of X-rays from a free-electron laser (FEL) (16-18). Figure 1 illustrates the experiment, in which ${ }^{4} \mathrm{He}$ droplets with radii $R=100-1000 \mathrm{~nm}\left(\mathrm{~N}_{\mathrm{He}}=10^{8}-10^{11}\right)$ were produced upon fragmentation of liquid helium expanding into vacuum $(15,19-20)$, see Section $\mathrm{S} 1$ of $(21)$. After a time of flight of $3.8 \mathrm{~ms}$ across a distance of $640 \mathrm{~mm}$ from the nozzle, the droplets traversed the focus of the FEL beam $(\mathrm{h} v=1.5 \mathrm{keV}, \lambda=0.827 \mathrm{~nm})$. Diffraction images were recorded using a pnCCD detector placed $\approx 565 \mathrm{~mm}$ behind the interaction volume. Each image originates from a single droplet irradiated by a single FEL shot. The low density core of ${ }^{4} \mathrm{He}$ vortices is $\approx 0.2 \mathrm{~nm}$ in diameter $(7)$, which does not provide sufficient contrast for direct detection by X-ray scattering. Therefore, the droplets are doped with $\mathrm{Xe}$ atoms $\left(\mathrm{N}_{\mathrm{Xe}} \approx 10^{-3} \cdot \mathrm{N}_{\mathrm{He}}\right)$, which cluster along the vortex cores $(10,15)$ and act as a contrast agent.

Figures 2, A-C show diffraction images of individual neat helium droplets. The circular and elliptical diffraction contours in Figs. $2 \mathrm{~A}$ and $2 \mathrm{~B}$ are consistent with diffraction from spheroidal droplets with a symmetry half axis, $\boldsymbol{a}$, and two equal perpendicular half axes, $\boldsymbol{b}$, (Fig. $\mathrm{S} 2)$. The aspect ratio, $A R=($ long half axis $) /($ short half axis $) \geq 1$, of the diffraction contours quantifies the distortion of the droplets from a spherical shape $(A R=1)$. Each diffraction image reflects the projection of a single droplet's density profile onto the detector plane; the longer axis in the diffraction pattern corresponds to the shorter droplet axis and vice versa. Because the $\boldsymbol{a}$ axis subtends an arbitrary angle with the X-ray beam, only the $\boldsymbol{b}$-axis and an upper boundary, $\boldsymbol{a} \leq$ $\boldsymbol{b} / A R$, can be deduced from each image. The pattern in Fig. 2A originates either from a spherical droplet with $R=\boldsymbol{a}=\boldsymbol{b}=(298 \pm 5) \mathrm{nm}$ or from a spheroidal droplet with $\boldsymbol{b}=R$ and its $\boldsymbol{a}$-axis aligned parallel to the X-ray beam. The diffraction pattern in Fig. 2B corresponds to a spheroid with $\boldsymbol{b}=(284 \pm 5) \mathrm{nm}$ and $\boldsymbol{a} \leq 0.87 \cdot \boldsymbol{b}=(247 \pm 5) \mathrm{nm}$ (Section S2 of (21)).

Surprisingly, approximately $1 \%$ of the diffraction images cannot be described by ellipses and exhibit very high aspect ratios of $1.7<A R<2.3$, such as $A R=1.92$ in Fig. 2C. Figure 2D shows the outline of the corresponding droplet, which was obtained by inverse Fourier transform (IFT) of Fig. 2C (Section S3 of (21)). The droplet is wheel-shaped with two nearly parallel surfaces and half axes $\boldsymbol{a}=(220 \pm 15) \mathrm{nm}$ and $\boldsymbol{b}=(422 \pm 10) \mathrm{nm}$. The intense diagonal streak in 
Fig. 2C indicates that the droplet was imaged edge-on within $\pm 5^{\circ}$ (see Section S3 of (21)). Thus, values for both $\boldsymbol{a}$ and $\boldsymbol{b}$ can be determined.

Our measurements reveal that, on average, approximately $40 \%$ of He droplets (Section S5 of (21)) in the beam are not spherical, as was previously assumed (20), but are better represented by spheroids or wheel shapes. In general, a droplet may acquire a non-spherical shape due to rotational or vibrational excitation. However, our estimates show that vibrational shape oscillations should decay before the interaction point (Section S6 of (21)). Therefore, the elliptical and streaked diffraction patterns are ascribed to oblate rotating droplets. The droplet rotation likely originates from inhomogeneous flow of helium through the nozzle during the expansion (15).

The shapes adopted by the rotating quantum droplets display similarities and pointed differences when compared with their classical counterparts. A classical droplet, rotating as a rigid body, can be described by the reduced angular velocity,

$$
\Omega=\sqrt{\frac{3 \cdot \rho \cdot V}{32 \cdot \pi \cdot \sigma}} \cdot \omega,
$$

which defines the droplet's aspect ratio (22-23). Here, $\rho$ is the density, $\sigma$ is the surface tension, and $V$ is the volume of the droplet. No droplet is stable beyond the disintegration limit of $\Omega_{\operatorname{MAX}}=$ 0.75 . At small $\Omega$, a droplet has a spheroidal shape. Beyond $\Omega=0.56(\boldsymbol{b} / \boldsymbol{a}=1.50)$ viscous classical droplets become unstable and begin to exhibit two-lobed shapes, resembling a peanut that rotates around its short axis. Multi-lobed droplet shapes emerge at even higher $\Omega$ (22-24). In this work, we observe axially symmetric droplets with aspect ratios as high as $\boldsymbol{b} / \boldsymbol{a}=2.3$, corresponding to $\Omega=0.71$ (Section S4 of (21)), which is considerably higher than the shape instability threshold of classical droplets. No evidence for multi-lobed shapes was detected. Our results confirm the predicted extended range of stability in rotating quantum liquids (23) and indicate that superfluid droplets remain axially symmetric up to rotational speeds close to $\Omega_{\mathrm{MAX}}$.

The angular velocities, $\omega$, of rotating droplets can be determined from the degree of centrifugal distortion, quantified by the $\boldsymbol{a}$ - and $\boldsymbol{b}$ - half axes (Section S4 of (21)) (23). For the image in Fig. 2C, this analysis leads to $\omega=1.4 \times 10^{7} \mathrm{~s}^{-1}$. The rotation of a superfluid may manifest as a lattice of uniformly distributed parallel vortices $(1,6-7,9)$ with an area density of:

$$
n_{V}=\frac{2 \omega \cdot M}{h}
$$

Here, $M$ is the mass of the ${ }^{4} \mathrm{He}$ atom, $h$ is Planck's constant, and $n_{V}$ is the number of vortices per unit area in a plane perpendicular to the axis of rotation (6-7). For the droplet imaged in Fig. 2C, Eq. 2 predicts a vortex density of $n_{V}=2.8 \times 10^{14} \mathrm{~m}^{-2}$ and a total number of vortices of $N_{V}=\pi \cdot b^{2} \cdot n_{V}=160$. Evidently, droplets in the beam are characterized by a substantial degree of rotational excitation, and thus should contain large numbers of quantum vortices. The existence of these vortices is confirmed by doping the He droplets with Xe atoms.

Figure 3 shows diffraction images of He droplets doped with Xe atoms. In addition to the characteristic ring patterns from the droplets, many images exhibit Bragg spots that either lie on a line crossing the image center (Fig. 3A) or form an equilateral triangular pattern (Fig. 3B). The Bragg spot separations in Fig. 3 correspond to regularly spaced Xe structures with periods of $d \approx$ $100 \mathrm{~nm}$, whereas the ring patterns arise from a droplet with $R \approx 1 \mu \mathrm{m}$. These numbers are consistent with the condensation of $\mathrm{Xe}$ atoms along the cores of multiple parallel vortices arranged in a lattice within the superfluid droplet (Fig. 3C). According to this model, both linear and triangular Bragg spot arrangements emerge from ordered lattices with different relative 
angles, $\Theta$, between the X-ray beam and the vortex lines. The actual shape of the vortices cannot be determined from the Bragg spots, although the vortices in the arrays are expected to have some curvature as they terminate perpendicular to the droplet's surface. Approximately $5 \%$ of the doped droplet images exhibit Bragg spots. Considering that the appearance of Bragg spots depends critically on the relative alignment of the vortex structures and the X-ray beam, which is randomly distributed in these experiments, we estimate that about $50 \%$ of droplets contain vortex lattices (Section S7 of (21)).

The identification of quantum vortices provides direct evidence of the superfluidity of He nanodroplets. The appearance of triangular vortex arrangements agrees with previous observations of triangular arrays of quantum vortices in rarified BECs (25-26). The diameters of the vortex cores in superfluid He, however, are small compared to the droplet sizes and the vortex length scales, which can lead to extended, three-dimensional vortex arrangements.

The diffraction pattern in Fig. 3B provides a direct measure of the vortex density, $n_{V}=$ $4.5 \times 10^{13} \mathrm{~m}^{-2}$, and the droplet radius, $\boldsymbol{b}=1100 \mathrm{~nm}$, corresponding to a total number of vortices $N_{V}=170$. The angular velocity of the rotating droplet is $\omega=2.2 \times 10^{6} \mathrm{~s}^{-1}$ (Eq. 2). The diffraction rings in Fig. 3B are circular within the experimental resolution $(\sim 3 \%)$. This observation and, in particular, the emergence of the triangular Bragg pattern, indicate that the droplet was imaged almost exactly along the $\boldsymbol{a}$ axis. From the angular velocity and the equatorial radius $\boldsymbol{b}$, the aspect ratio and reduced angular velocity of the droplet are estimated to be $A R=1.34$ and $\Omega=0.50$, respectively (Section S4 of (21)). These values fall well within the axisymmetric shape stability limits for rotating droplets. The vortex density in this droplet is about five orders of magnitude larger than previously observed in rotating bucket experiments with bulk superfluid helium (910). These numbers demonstrate that superfluid He droplets provide access to unexplored regimes of rotational excitation in quantum liquids. It is intriguing that, although observation of the wheel shapes in smaller droplets $(b \approx 300-400 \mathrm{~nm})$ indicates the existence of high vortex densities in the range of $n_{V} \approx 3 \times 10^{14} \mathrm{~m}^{-2}$, no corresponding Bragg patterns were observed in these droplets. (Section S7 of (21)) This may indicate that vortices at extremely high densities fail to crystallize and instead form a disordered state with little resemblance to a lattice. Another possibility is the existence of non-equilibrium states, which may be related to quantum turbulence. However, estimates (Section S6 of (21)) show that turbulence, which accompanies establishment (27) or breakdown (28) of equilibrium quantum rotation, decays before the interaction point. Hydrodynamic instability of the droplet shape at high angular velocities may also disrupt vortex arrays. The possibility for the formation of non-stationary vortex states in superfluid helium has been discussed ( 6 ) but has never been confirmed experimentally. In addition, BECs at high $\omega$ are predicted to undergo a quantum phase transition into a highly correlated non-superfluid state devoid of any vortices (26). It would therefore be interesting to explore whether similar concepts apply to rotating He droplets at high $\omega$. 


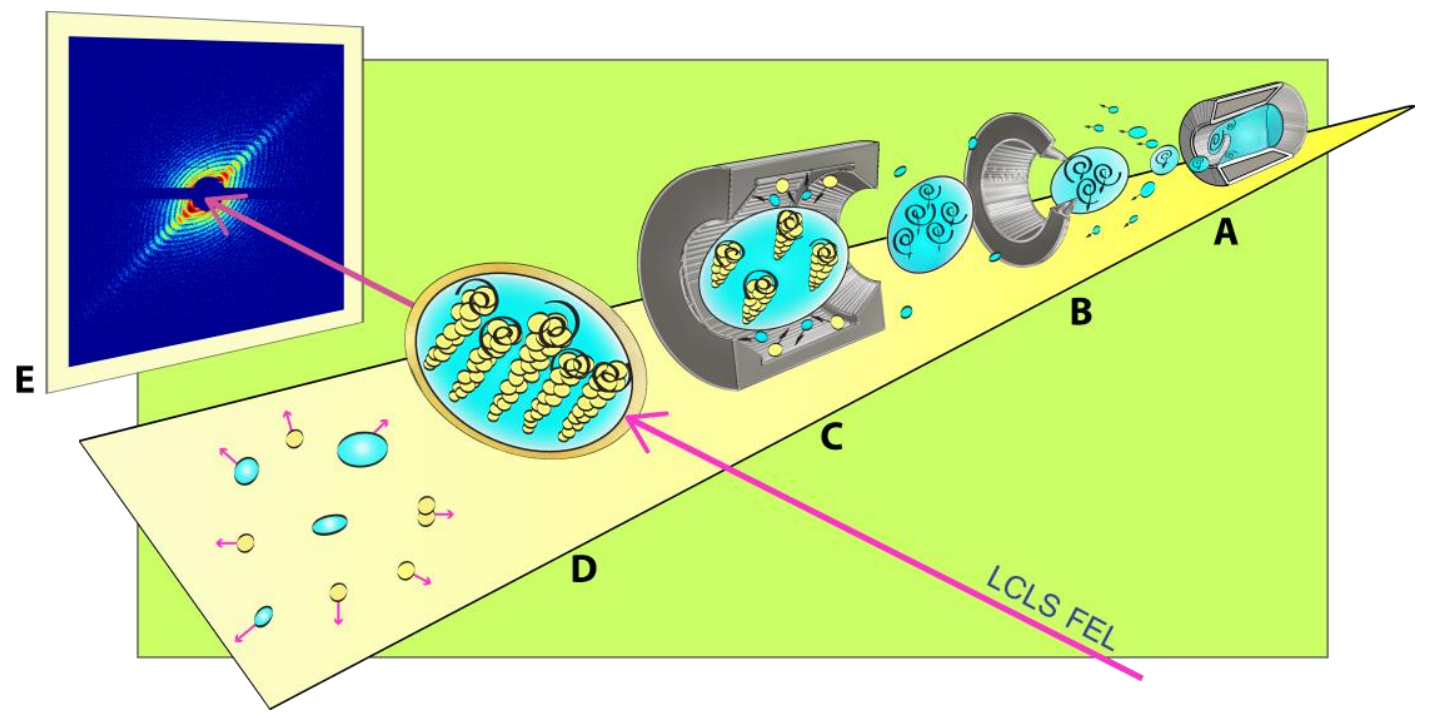

Fig. 1. Experimental setup.

(A) Rotating droplets are produced by expanding He fluid into vacuum through a $5 \mu \mathrm{m}$ nozzle at a temperature of $T_{0} \approx 5 \mathrm{~K}$ and a backing pressure $P_{0}=20 \mathrm{bar}$; (B) quantum vortices form upon evaporative cooling of rotating droplets to below $T_{\lambda} ;(\mathbf{C})$ droplets are doped with Xe atoms in a cell filled with Xe gas; (D, E) X-ray diffraction images of single droplets are recorded using single FEL light pulses. 

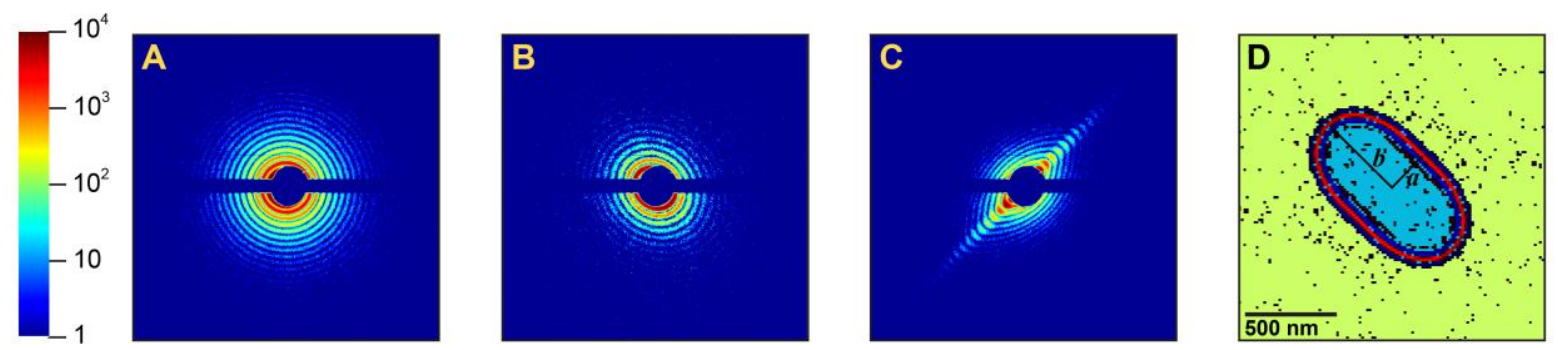

E

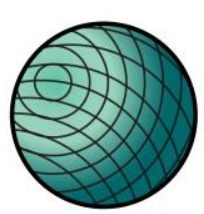

$\mathbf{F}$

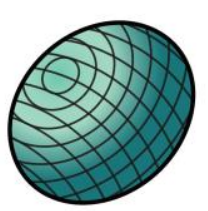

G

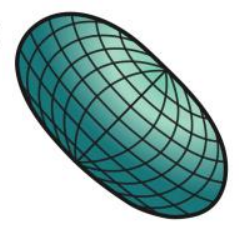

Fig. 2. X-ray diffraction images of neat He droplets.

(A), (B), and (C) represent circular, elliptical, and streaked patterns, respectively, displayed in a logarithmic color scale. 3D representations of the droplet shapes have been placed below the corresponding images (E-G). (D) shows a droplet outline reconstructed from the diffraction pattern in (C) by IFT. Note that the droplet itself is not hollow but filled. 

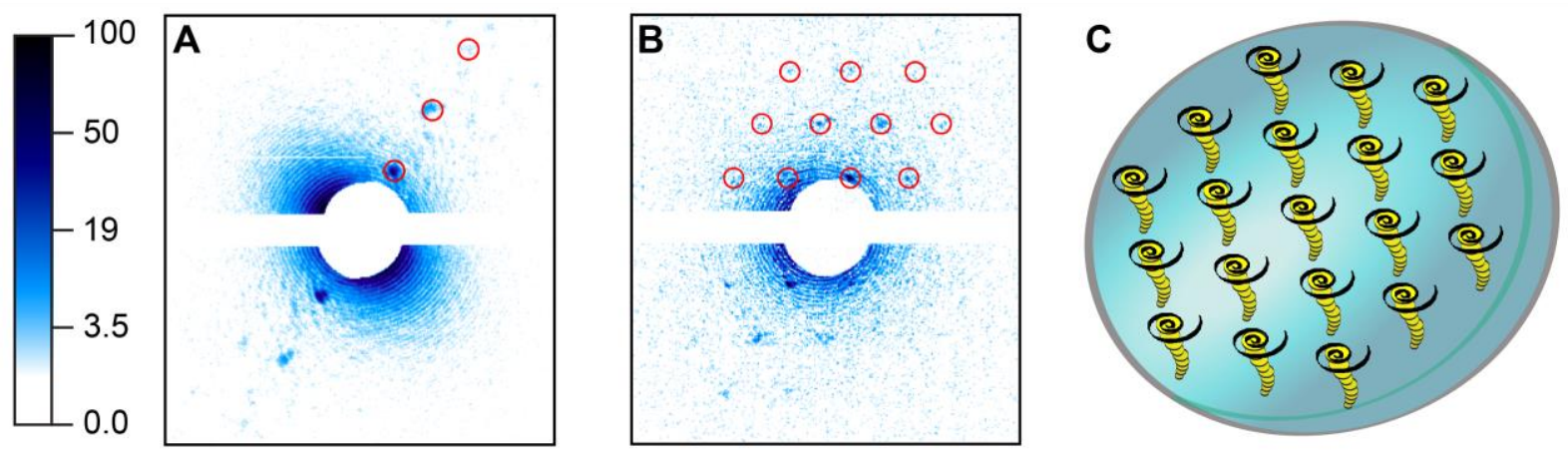

\section{Fig. 3. He droplets doped with Xe atoms.}

(A and B) show X-ray diffraction images of doped droplets, displayed in a logarithmic intensity scale. (C) depicts the droplet and embedded Xe clusters. (A) and (B) correspond to tilted and parallel alignments of the vortex axes with respect to the incident X-ray beam, respectively. 


\section{Supplementary Materials:}

\section{Materials and Methods}

\section{S1. Experimental Technique}

The He droplet molecular beam experiment has been described previously $(20,29)$. Figure S1 shows a schematic of the vacuum apparatus employed in this work, in which helium nanodroplets form upon expansion of high purity $(99.9999 \%)$ He into vacuum through a nozzle (NZ) with a $5 \mu \mathrm{m}$ nominal diameter and a $2 \mu \mathrm{m}$ nominal channel length, at a temperature of $5 \mathrm{~K}$ and a backing pressure of 20 bar. In this work, two different nozzle plates were used to produce droplets containing average numbers of $\mathrm{He}$ atoms of $\left\langle N_{\mathrm{He}}\right\rangle=3 \times 10^{9}$ and $\left\langle N_{\mathrm{He}}\right\rangle=9 \times 10^{10}$, corresponding to average radii of $320 \mathrm{~nm}$ and $1000 \mathrm{~nm}$, respectively. Average droplet sizes were determined by titrating the droplets with Xe atoms using a procedure described in ref. (19). Some measurements reported in Figs. S5 and S6 were also performed at higher nozzle temperatures.

For the experiments described herein, the central part of the He beam expansion was directed through a $0.5 \mathrm{~mm}$ diameter skimmer (SK) and the resulting droplet beam was doped with Xe atoms in a $10 \mathrm{~cm}$ long pickup cell (PC) placed $15 \mathrm{~cm}$ from the droplet source. The degree of Xe doping was controlled by varying the Xe pressure in the pickup cell, which was measured in absolute units by a membrane manometer. The droplet beam then crossed the 25 $\mu \mathrm{m}^{2}$ focus of the X-ray beam from the free-electron laser (FEL) at the interaction point (IP) 640 $\mathrm{mm}$ away from the He nozzle. The FEL was operated at $\mathrm{h} v=1.5 \mathrm{keV}(\lambda=0.827 \mathrm{~nm})$ and delivered approximately $10^{12}$ photons per 100 fs pulse at a repetition rate of $120 \mathrm{~Hz}$. The experiments were performed using the CFEL-ASG Multi-Purpose (CAMP) instrument at the Atomic, Molecular, and Optical Science (AMO) beamline of the Linac Coherent Light Source (LCLS); for details, see refs. (16-18). Scattered X-rays were detected at small scattering angles (<0.05 rad) using a cooled pnCCD detector with approximately $10^{6}$ pixels $\left(75 \times 75 \mu \mathrm{m}^{2} /\right.$ pixel $)$. The detector consists of two panels $\left(75 \times 37 \mathrm{~mm}^{2}\right.$ each) separated by about $2 \mathrm{~mm}$ and placed perpendicular to the X-ray beam at distances of $564 \mathrm{~mm}$ and $567 \mathrm{~mm}$ behind the scattering center (see Fig. S2). Both panels have semicircular cuts to accommodate the primary X-ray beam, which crossed the detector approximately in its geometric center. One detected X-ray photon results in about 35 units of intensity, as shown by the color scale in Fig. 2 of the main article.

The probability for a droplet to reside in the FEL focal volume during a laser pulse was estimated to be less than $\approx 10^{-3}$, using the measured droplet flux (19). This estimate agrees with the observed hit rate. Therefore, the probability of finding two droplets in the detection volume is negligible $\left(\leq 10^{-6}\right)$ and each diffraction image originates from a single droplet irradiated by a single FEL shot. In addition to scattering from He and Xe atoms, many X-ray photons within a single FEL shot are absorbed, leading to ionization and, ultimately, disintegration of the droplets via Coulomb explosion. The increase in the radius of a droplet due to Coulomb explosion (30) during an FEL pulse was estimated to be less than $1 \%$, which is within the uncertainty of droplet size determinations in this work. For each hit, the resulting ion distribution was measured using a time-of-flight (TOF) mass spectrometer, confirming the purity of the droplets. Therefore, the obtained diffraction images represent the shapes of intact, single He droplets in the beam. 
After passing through the scattering chamber, the droplet beam entered the beam dump vacuum chamber, where the beam intensity was monitored via the partial He pressure, $P_{\text {He. }}$ The average number of Xe atoms captured per He droplet, $\left\langle N_{X e}\right\rangle$, as well as the average droplet size before doping, $\left\langle N_{H e}\right\rangle$, were estimated by measuring the attenuation of the droplet beam due to dopant-induced evaporation of $\mathrm{He}$ atoms (19). Upon repeated capture of $\mathrm{Xe}$ atoms and concomitant evaporation of $\mathrm{He}$ atoms, $\left\langle N_{H e}\right\rangle$ decreases, as monitored by a reduction in the average partial pressure of He in the dump chamber, $\Delta P_{\mathrm{He}}$. The energy absorbed by the droplet upon capture of Xe atoms primarily arises from the binding energy released by the formation of $\mathrm{Xe}_{N}$ clusters and the kinetic energy of room-temperature Xe atoms in the pickup cell. Thus $\left\langle N_{X e}\right\rangle$ can be estimated by

$$
\left\langle N_{\mathrm{Xe}}\right\rangle=\frac{\Delta P_{\mathrm{He}}\left\langle N_{\mathrm{He}}\right\rangle}{P_{\mathrm{He}}} \cdot \frac{E_{\mathrm{He}}}{E_{\mathrm{Xe}}},
$$

where $E_{\mathrm{He}}$ is the $0.6 \mathrm{meV}$ (31) binding energy of He atoms to the droplet, $\left\langle N_{H e}\right\rangle$ is the initial average size of the He droplet, and $E_{\mathrm{Xe}} \approx 0.15 \mathrm{eV}$ is the average total energy released upon capture and condensation of one Xe atom. In Fig 3 of the main article, He droplets were doped with Xe atoms until about $5 \%$ of the He atoms were evaporated. This corresponds to a droplet radius reduction of approximately $2 \%$. The doped droplets contained a factor of about 5000 more He then Xe atoms.

As He droplets capture Xe atoms in the pickup cell, their temperature increases above the minimum temperature they reach in vacuum $(T=0.37 \mathrm{~K}(32))$. This temperature rise was estimated $(19,31)$ using the known temperature-dependence of the vapor pressure of $\mathrm{He}(33)$ and the rate of evaporation of He atoms from the droplet during a time-of-flight through the pickup cell of about $600 \mu \mathrm{s}$. For all experiments carried out in this work, the droplet temperature in the pickup cell did not exceed $0.8 \mathrm{~K}$ and remained well below the superfluid transition temperature of $2.17 \mathrm{~K}$. 


\section{Supplementary Text}

\section{S2. Scattering of X-Rays by Spheroids}

\section{S2.1. Rayleigh-Gans Theory for Small Angle Scattering}

Figure S2 shows the geometry of the FEL X-ray beam scattered by a spheroidal droplet. For an optically thin object, each of its volume elements can be considered as an independent Rayleigh scattering center for the incident wave (34), which constitutes the so-called RayleighGans approximation. The total scattering amplitude is described by the superposition of scattering waves from all infinitesimal volume elements of the droplet. The amplitude of the scattered wave is given by Eq. (8.1.1) in ref. (34):

$$
S(\theta)=\frac{3}{4 \pi} k^{3}\left(\frac{n^{2}-1}{n^{2}+2}\right) \cdot \int_{V} e^{i(\bar{q} \cdot \vec{d})} d \tau .
$$

Here, $e^{i(\vec{q} \cdot \vec{d})}$ accounts for the phase difference between wavelets originating from different volume elements with real-space coordinates $\vec{d} ; n$ is the complex refractive index of the droplet; $k=|\vec{k}|$ is the wave number of the incident wave; and $\vec{q}$ is the change of the wave vector upon elastic scattering, $|\vec{q}|=2 k \sin (\theta / 2)$, in which $\theta$ is the scattering angle.

The Rayleigh-Gans approximation holds if $\frac{2 \cdot R \cdot|n-1|}{\lambda}<<1(\lambda$ : wavelength of light, $\mathrm{R}$ : typical radius of scattering object). The refractive index of liquid He at $h v=1.5 \mathrm{KeV}(\lambda=0.827$ $\mathrm{nm})$ is given by $n=\left(1-1.34 \times 10^{-5}+1.6 \times 10^{-8} i\right) \approx 1$. Thus, the Rayleigh-Gans approximation is well satisfied for the largest $\mathrm{He}$ droplets in this work, with $\mathrm{R} \approx 1500 \mathrm{~nm}$, for which $\frac{2 \cdot R \cdot|n-1|}{\lambda} \approx 0.05$. Consequently, the intensity of the scattered light can be obtained from:

$$
I(\theta)=\frac{|S(\theta)|^{2}}{k^{2} \cdot r^{2}} \cdot I_{0}=\left(\frac{k^{4} \cdot V^{2}}{4 \pi^{2} \cdot r^{2}}\right) \cdot|n-1|^{2} \cdot P(\theta) \cdot I_{0} .
$$

In Eq. (S2.2), $r$ is the distance between the scattering center and the detector, $V$ is the volume of the droplet (Eq. 8.1.5 in ref. (34)), and $I_{0}$ is the incident photon flux. $P(\theta)$ is the form factor ((34), Eq. 8.1.6):

$$
P(\theta)=\frac{1}{V^{2}}\left|\int_{V} e^{i(\bar{q} \cdot \vec{d})} d \tau\right|^{2} .
$$

\section{S2.2. Spherical Droplet}

For a spherical droplet of radius, $R$, the form factor is:

$$
P_{\text {sphere }}(\theta)=\frac{9 \pi}{2} \frac{\left(J_{3 / 2}(q R)\right)^{2}}{(q R)^{3}},
$$

in which $J_{3 / 2}$ is the Bessel function of the order of $3 / 2((34)$, Table 8.5, p 482). Substituting Eq. (S2.4) to Eq. (S2.2) and taking $V=V_{\text {sphere }}=\frac{4 \pi}{3} R^{3}$ the scattering intensity is: 


$$
I_{\text {sphere }}(\theta)=2 \pi \cdot\left(\frac{k^{4} \cdot R^{3}}{r^{2}}\right) \cdot|n-1|^{2} \cdot \frac{\left(J_{3 / 2}(q R)\right)^{2}}{q^{3}} \cdot I_{0} \text {. }
$$

\section{S2.3. Spheroidal Droplets}

The form factor of a spheroid with symmetry half axis, $\boldsymbol{a}$, and two equal half axes, $\boldsymbol{b}$, (see Fig. S2) is also expressed in terms of the Bessel function of the order of $3 / 2$ as:

$$
P_{\text {spheroid }}(\theta)=\frac{9 \pi}{2} \frac{\left(J_{3 / 2}(u)\right)^{2}}{(u)^{3}},
$$

in which $u=q \cdot R_{\text {eff }}\left(a, b, \Theta, \Phi, \Phi_{0}\right)((34)$, Table 8.5 p.484). In the limit of small angle scattering, the effective radius, $R_{\text {eff }}$, is given by (35):

$$
R_{e f f}\left(a, b, \Theta, \Phi, \Phi_{0}\right)=b \sqrt{\left(\cos ^{2}(\Theta)+\frac{a^{2}}{b^{2}} \sin ^{2}(\Theta)\right) \cos ^{2}\left(\Phi-\Phi_{0}\right)+\sin ^{2}\left(\Phi-\Phi_{0}\right)},
$$

in which $\Theta$ is the angle between the symmetry axis $\boldsymbol{a}$ and the X-ray beam, while $\Phi$ and $\Phi_{0}$ define the azimuthal angles of $q$ and of the projection of $\boldsymbol{a}$ in the scattering plane, respectively, as shown in Fig. S2. Following from Eq. (S2.7), the aspect ratio, A/B, of the elliptical diffraction image is given by Eq. (S2.8),

$$
\frac{A}{B}=\left[\sqrt{\cos ^{2}(\Theta)+\frac{a^{2}}{b^{2}} \sin ^{2}(\Theta)}\right]^{-1},
$$

in which $\mathrm{A}$ and $\mathrm{B}$ are the principal image axes. Accordingly, $\mathrm{A} / \mathrm{B}>1$ for oblate spheroids and $\mathrm{A} / \mathrm{B}<1$ for prolate spheroids.

Equation (S2.7) shows that only the angle $\Phi_{0}$ or $\left(\Phi_{0}+\pi / 2\right)$ and the half axes $\boldsymbol{b}$ (which do not contain the symmetry axes) can be determined from the elliptical diffraction pattern. In contrast, the symmetry half axis, $\boldsymbol{a}$, and the angle, $\Theta$, remain interrelated. Therefore, the observed elliptic patterns are consistent with both oblate and prolate droplets. For example, the pattern in Fig. 2B of the article can be described by $\left(\boldsymbol{b}, \Phi_{0}\right)=(284 \pm 5 \mathrm{~nm}, 0.79 \mathrm{rad})$ or $(247 \pm 5$ $\mathrm{nm}, 2.36 \mathrm{rad}$ ) and may originate from either an oblate or a prolate spheroidal droplet with $\sqrt{b^{2} \cos ^{2}(\Theta)+a^{2} \sin ^{2}(\Theta)}=(247 \pm 5) \mathrm{nm}$ or $(284 \pm 5) \mathrm{nm}$, respectively. Since $\Theta$ remains undefined, it follows that only boundary values can be obtained for the symmetry axis $\boldsymbol{a}: \boldsymbol{a} \leq 247$ $\mathrm{nm}$ or $\boldsymbol{a} \geq 284 \mathrm{~nm}$, respectively. In order to estimate the values of $\boldsymbol{b}$ and $\sqrt{b^{2} \cos ^{2}(\Theta)+a^{2} \sin ^{2}(\Theta)}$, the observed diffraction patterns were fitted with Eqs. (S2.6-S2.7) using a non-linear least squares procedure.

\section{S3. Wheel-Shaped Droplets}

Diffraction patterns with streaks, as shown in Fig. 2C of the main article, cannot be fitted by ellipses. Streaks are not expected for ellipsoidal bodies of any aspect ratio; their presence indicates diffraction from a wheel-shaped droplet with two (nearly) parallel surfaces. In lieu of an analytical expression for diffraction from such shapes, we used the inverse Fourier transform (IFT) of the square root of the intensity in Fig 2C to obtain the droplet shape. In order to reconstruct the phase information, we assumed that the droplet was centro-symmetric, as per a classical rotating droplet (22), and was characterized by a real-valued scattering amplitude. Accordingly, we assigned alternating (+/-) signs for the scattering amplitudes of consecutive 
diffraction maxima in Fig. 2C. The outline of a diffracting droplet that has two nearly parallel surfaces is shown in Fig. 2D of the main article. The droplet density profile cannot be obtained via IFT from the available data due to the loss of low frequency information in the central hole of the detector. The pronounced diagonal streak in the diffraction pattern (Fig. 2C) indicates that the wheel shape was imaged edge-on within about \pm 5 degrees. This tolerance was estimated by calculating diffraction patterns from a wheel shaped droplet for different angles between the droplet axis $\boldsymbol{a}$ and the X-ray beam. At relative angles beyond $\approx 90 \pm 5^{\circ}$, wheel-shaped droplets will produce nearly elliptical diffraction images devoid of any streaks. Therefore, in contrast to the case of spheroidal droplets, absolute values for both $\boldsymbol{a}$ - and $\boldsymbol{b}$-axes can be determined for droplet images that exhibit pronounced streaks. The two half axes of the wheel-shaped droplet were derived from the average distance between the consecutive diffraction maxima along the major and minor axes of the diffraction image and were found to be $\boldsymbol{a}=(220 \pm 15) \mathrm{nm}$ and $\boldsymbol{b}=$ $(422 \pm 10) \mathrm{nm}$, respectively. These values agree well with those obtained directly from the IFT in Fig. $2 \mathrm{D}(\boldsymbol{a} \approx 215 \mathrm{~nm}, \boldsymbol{b} \approx 430 \mathrm{~nm})$.

\section{S4. Centrifugal Distortion in Rotating Droplets}

The shape of a classical droplet executing rigid body rotation is defined by the balance between the surface tension of the curved droplet and the centrifugal force. According to ref. (23), an axially symmetric, rotating droplet can be described by a dimensionless rotational number, $\Sigma$, which determines the shape of the droplet at angular velocity $\omega$ :

$$
\Sigma=\frac{\rho \cdot \omega^{2} \cdot b^{3}}{8 \cdot \sigma} .
$$

In Eq. (S4.1), $\rho=145 \mathrm{~kg} / \mathrm{m}^{3}$ is the density of liquid helium, $\boldsymbol{b}$ is the equatorial radius of the droplet, and $\sigma=0.354 \times 10^{-3} \mathrm{~N} / \mathrm{m}$ is the surface tension of liquid helium (33). The droplet has a spherical shape at $\Sigma=0$ and is a spheroid at small $\Sigma$. As $\Sigma$ increases, the figure rapidly departs from the spheroidal form: at $\Sigma=1$ the droplet adopts a wheel shape with flat poles; at $\Sigma>1$ the droplet develops a dimple that deepens quickly and reduces the polar droplet diameter to zero at $\Sigma=2.33$ (23). The shape of a rotating, axially symmetric droplet is given, in terms of $\Sigma$, by Eq. (A16) in ref. (23). A related quantity, the bond number, $\Omega$, is used in the more recent literature (22):

$$
\Omega=\sqrt{\frac{3 \cdot \rho \cdot V}{32 \cdot \pi \cdot \sigma}} \cdot \omega,
$$

Here, $V$ is the volume of the droplet. No droplet is stable beyond the disintegration limit of $\Omega_{\text {MAX }}$ $=0.75$. This equation was used to obtain $\Omega$ and $\Sigma$ for droplets as a function of their aspect ratios, $\boldsymbol{b} / \boldsymbol{a}$, as illustrated in Figs S3A and S3B, respectively. These functions were combined with Eq. (S4.1) to calculate the droplets' angular velocities from $\Sigma$ and the absolute value of $\boldsymbol{b}$. Cross sections of representative droplet shapes are shown in Fig. S4. The shapes of rotating superfluid droplets in equilibrium are expected to resemble classical droplets rotating at the same $\omega$ due to similar velocity fields far from the vortex cores. For example, the shape of a rotating superfluid in a cylindrical container adopts a parabolic shape similar to that of a classically rotating liquid $(2,7)$. In principle, deviations from equilibrium conditions, such as a radially varying vorticity, may limit the applicability of Eqs. S4.1 and S4.2. The observation of Bragg spots, however, suggests that equilibrium can be established within the time-of-flight of the droplets before they reach the interaction volume. 


\section{S5. Fraction of Non-Spherical Droplets}

The aspect ratio, $A R$, is defined as the ratio of the semi-major axis and the semi-minor axis of a droplet's projection onto the detector plane. The one-sigma precision of the axis determination is $\sim 2 \%$, resulting in a $\sim 3 \%$ precision for $A R$. We define non-spherical droplets as those whose aspect ratios deviate from $A R=1$ by at least two sigma $(A R \geq 1.06)$. Figure S5 shows the fraction of non-spherical droplets at different average droplet sizes, obtained from the semi-major axis of the droplet's projection onto the detector. The results are based on the analysis of 663 diffraction images of neat He droplets with radii $\mathrm{R} \leq 600 \mathrm{~nm}$ generated under various conditions. Note that the ratios shown in Fig. S5 are lower bounds for the fractions of non-spherical droplets, since spheroids with symmetry axes aligned perpendicular to the detector will cast circular diffraction patterns independent of their aspect ratios.

\section{S6. Relaxation Time of Different Excitations in He droplets}

The relaxation times of different types of droplet excitations depend on the droplet's temperature. After formation close to the nozzle, a droplet moving at $\approx 170 \mathrm{~m} / \mathrm{s}$ encounters four distinct regions during its flight toward the interaction point (Fig. S1):

Region 1 The distance between the nozzle and the first skimmer $(x \approx 20 \mathrm{~mm})$, which the droplet traverses within $\mathrm{t} \approx 120 \mu \mathrm{s}$;

Region 2 The region inside the skimmer chamber excluding the pickup cell, $x=370 \mathrm{~mm}$, $\mathrm{t}=2.2 \mathrm{~ms}, \mathrm{P}=6 \times 10^{-4} \mathrm{mbar}$;

Region 3 The region inside the pickup cell, $x=100 \mathrm{~mm}, \mathrm{t}=0.6 \mathrm{~ms}$;

Region 4 The region inside the scattering chamber before the interaction region, $x=150 \mathrm{~mm}$, $\mathrm{t}=0.9 \mathrm{~ms}, \mathrm{P}=10^{-6}$ mbar.

The droplet temperature in Region 1 can be estimated from the local pressure, assuming the droplet temperature is defined by the saturated vapor pressure (33). The local pressure in the nozzle chamber is estimated to be about $10^{-2}$ mbar. Therefore, in Region 1, the temperature drops from $5 \mathrm{~K}$ close to the nozzle to $\sim 0.9 \mathrm{~K}$ at the skimmer. The temperatures $\mathrm{T}_{2}$ and $\mathrm{T}_{4}$ in Regions 2 and 4, respectively, are estimated from the collision rate (19) to be $0.7 \mathrm{~K}$ and $0.4 \mathrm{~K}$, respectively. Finally, during the doping in the pickup cell, the temperature of the droplets increases to $\mathrm{T}_{3} \sim 0.8$ $\mathrm{K}$.

\section{S6.1. Droplet Shape Oscillations}

The lifetime of the shape oscillations in a viscous droplet of radius, $\mathrm{R}$, having kinematic viscosity, $v$, is given by (36):

$$
\tau_{S O}=\frac{R^{2}}{(2 l+1)(l-1) \cdot v},
$$

where $l$ is the order of the excitation and $l=2$ represents the lowest quadrupolar droplet excitation. Equation (S6.1) holds when the mean free path of the droplet's elementary excitations, $\lambda$, is smaller than the droplet's radius. For a representative droplet in this work, with $\mathrm{R}=500 \mathrm{~nm}$, Eq. (S6.1) is valid at $\mathrm{T}>0.9 \mathrm{~K}$. At lower temperatures, the mean free path of the elementary excitations grows larger than the droplet size and Eq. (S6.1) should be replaced by one applicable to the molecular dissipation regime. It is clear that Eq. (S6.1) applies to Region 1. Calculations at $\mathrm{T}=0.9,1.5$ and $3.0 \mathrm{~K}\left(\mathrm{v}=4.3 \times 10^{-8}, 9.3 \times 10^{-9}\right.$ and $2.5 \times 10^{-8} \mathrm{~m}^{2} / \mathrm{s}$, respectively (33)) give $\tau_{S O}=1.2 \times 10^{-6}, 5.4 \times 10^{-6}$ and $2.0 \times 10^{-6} \mathrm{~s}$, respectively. These results are in good agreement with experimentally determined lifetimes of quadrupole excitations in magnetically levitated $\mathrm{He}$ droplets with $R=2.3 \mathrm{~mm}$ at $\mathrm{T}=0.65-2 \mathrm{~K}$ upon appropriate $R^{2}$ scaling (37). 
Therefore, we conclude that the shape oscillations decay much faster than the flight time through Region 1 of about $10^{-4} \mathrm{~s}$. The large fraction of nearly spherical $(A R<1.06)$ droplets in the beam provides additional support for fast quenching of shape oscillations. Droplet generation in the breakdown of a liquid jet (19) most likely leads to a substantial degree of shape oscillations in the vast majority of droplets near the nozzle. These oscillations must decay before the interaction region because the majority of observed droplets are spherical (see Fig. S5). Therefore, we ascribe all deformations to oblate rotating droplets.

\section{S6.2. Vortex Dynamics}

It is likely that vortices form in rotating droplets upon cooling to temperatures below $\mathrm{T}_{\lambda}$, although neither the point along the expansion line where the vortices nucleate nor the corresponding temperature of the droplets is known. Such a transition may proceed via an intermediate turbulent state (27-28). Excitations may also take the form of Kelvin waves on the vortices as well as vortex density waves.

The lifetime of vortex Kelvin waves of wavenumber $k$ due to interaction with thermal elementary excitations is given by (38)

$$
\tau_{K W}=\frac{4 \pi \rho_{s}}{k^{2} \gamma \cdot \ln \left(\frac{2}{k a}\right)},
$$

where $\rho_{s}$ is the superfluid density, $\gamma$ is the temperature dependent mutual friction coefficient (39) and $a \approx 0.1 \mathrm{~nm}$ is the radius of the vortex core. Taking the smallest possible wavenumber corresponding to a wavelength twice the droplet's diameter, $k=\pi / 2 R$, then at $\mathrm{T}=0.5,0.9$ and $1.5 \mathrm{~K}$, the lifetimes are $\tau_{K W}=2.3,9 \times 10^{-3}$ and $2.5 \times 10^{-5} \mathrm{~s}$, respectively. The relaxation time increases rapidly with decreasing temperature and exceeds the droplet time of flight of $\sim 4 \mathrm{~ms}$ near $1 \mathrm{~K}$. Therefore, Kelvin waves with long wavelengths may persist into the interaction region, distorting the shape of the vortices from equilibrium. The termination of vortices at the droplet's surface may lead to relaxation due to interaction with surface ripplons, thus shortening this lifetime. However the corresponding mutual friction coefficients remain unknown.

Relaxation times of density waves in the vortex lattice, $\tau_{D W}$, also affect the droplet dynamics. For a breathing mode of a vortex lattice in a cylinder filled with superfluid $(2,7)$, the lifetime is given by:

$$
\tau_{D W} \approx \frac{\kappa \cdot \rho_{s}}{2 \omega \cdot \gamma},
$$

where $\kappa=h / M$ is the quantum of circulation and $\omega$ is the angular velocity. Taking $\omega=10^{7} \mathrm{~s}^{-1}$, at $\mathrm{T}=0.5,0.9$ and $1.5 \mathrm{~K}$, Eq. (S6.3) gives $\tau_{D W}=0.07,3 \times 10^{-4}$ and $8 \times 10^{-7} \mathrm{~s}$, respectively. The relaxation time is, therefore, fast compared to the flight times in the expansion region and slow in the high vacuum regimes.

The establishment of quantum rotation via formation of a vortex array may proceed through a stage of quantum turbulence characterized by chaotic motion of vortex lines in the velocity fields of the other lines (27-28). Numerous experiments have demonstrated that the mean square vorticity, L, defined as the average length of vortex lines per unit volume, decays at long times ( $\mathrm{t}>>0)$ according to Eq. (S6.4) (28): 


$$
L=\frac{d}{2 \pi \kappa} \sqrt{\frac{27 C^{3}}{v}} t^{-3 / 2}
$$

In this expression, $d$ is the characteristic size of the system, usually associated with the container diameter for experiments using bulk LHe, taken here to be the droplet diameter, and $\mathrm{C}$ is the Kolmogorov constant usually estimated to be $\mathrm{C} \approx 1.5$. From a recent study of quantum turbulence at temperatures down to $0.08 \mathrm{~K}(28)$, Eq. (S6.4) was found to be still valid. However, the effective kinematic viscosity coefficient at $\mathrm{T}<0.8 \mathrm{~K}$ is $\approx 3 \times 10^{-3} \mathrm{~m}^{2} / \mathrm{s}$, a factor of 30 less than at $\mathrm{T}>1 \mathrm{~K}$. This effect was attributed to dissipation of small wavelength Kelvin waves by emission of phonons at low temperatures. Equation (S6.4) shows that the length of the vortex lines in turbulent flow drops by a factor of 1000 between $10^{-5} \mathrm{~s}$ and $10^{-3} \mathrm{~s}$.

From the above discussion we conclude that initial turbulence in the beam, which may affect the superfluid transition in rotating droplets and the formation of vortex lines, decays before the interaction point. However, the vortices may still contain some degree of Kelvin wave and density wave excitations. The corresponding degrees of these excitations cannot be estimated because the nucleation point of the vortices along the cooling curve is unknown. Even so, the observation of a hexagonal vortex lattice, as in Fig. 3B, indicates that droplet excitations have sufficiently relaxed for the formation of vortex lattices to occur.

\section{S7. Fraction of Large Droplets with Vortex Lattices}

In general, the Bragg spots are found by determining where the surface of an Ewald sphere, having in our case a radius of $K_{E}=2 \pi / \lambda=7.60 \mathrm{~nm}^{-1}$, intersects the reciprocal lattice structure, which is related to the direct space structure by Fourier transform. The reciprocal lattice of a hexagonal vortex lattice is approximated by a two dimensional hexagonal lattice. The experimental images correspond to intersections at different polar, $\theta$, and azimuthal, $\varphi$, angles, defined by the vortex array orientation with respect to the incoming X-ray beam. In the case of a hexagonal reciprocal lattice, a hexagonal diffraction pattern will be obtained if the X-ray beam impinges perpendicular to the reciprocal lattice plane at $\theta=0$, such as observed in Fig. 3B. The intensity of the Bragg spots decreases upon increase of the scattering angle due to the effect of the curvature of the Ewald sphere and the finite size of the diffracting Xe clusters trapped inside vortices. At $\theta \neq 0$, a series of intense spots lying approximately on a line arises at some favorable values of $\varphi$, such as in Fig. 3A. Finally, at the majority of $\theta$ and $\varphi$ values, the Ewald sphere does not cut through any dots in the reciprocal lattice and no Bragg spots are observed. Using arbitrary values of $\theta$ and $\varphi$, and assuming that a droplet contains a hexagonal array of 100 vortices, the probability for obtaining a hexagonal pattern is estimated to be about $10^{-3}$, whereas a linear pattern has a probability of about 0.1 , in agreement with its much more frequent occurrence.

In total, approximately $5 \%$ of the images of doped droplets exhibit Bragg spots, suggesting that approximately $50 \%$ of all droplets contain Xe cluster arrays and therefore vortex lattices. These results are based on the observation of 2030 diffraction images of doped droplets.

\section{S8. Appearance of Bragg Spots.}

Figure S6 shows a distribution of observed Bragg plane densities. The plot results from analyzing 69 diffraction images obtained from doped He droplets, for which both the separation between Bragg planes and the droplets' radii could be determined from the diffraction rings, such as in Figures 3A and 3B of the main article. In addition, some images contained clear Bragg 
spots, but the droplet diffraction rings could not be resolved due to low intensity or insufficient spatial resolution of the detector. The value $\eta=\sqrt{3} /\left(2 l^{2}\right)$ is plotted against the average droplet radius, where $l$ is the distance between Bragg planes. For the $(1,1)$ Bragg planes in equilateral triangular lattices, $\eta$ is equal to the vortex density, $n_{V}$. However, because the overwhelming majority of Bragg spots appeared as in Fig. 3A and could not be indexed, the value of $\eta$ represents an upper boundary for $n_{V}$. 


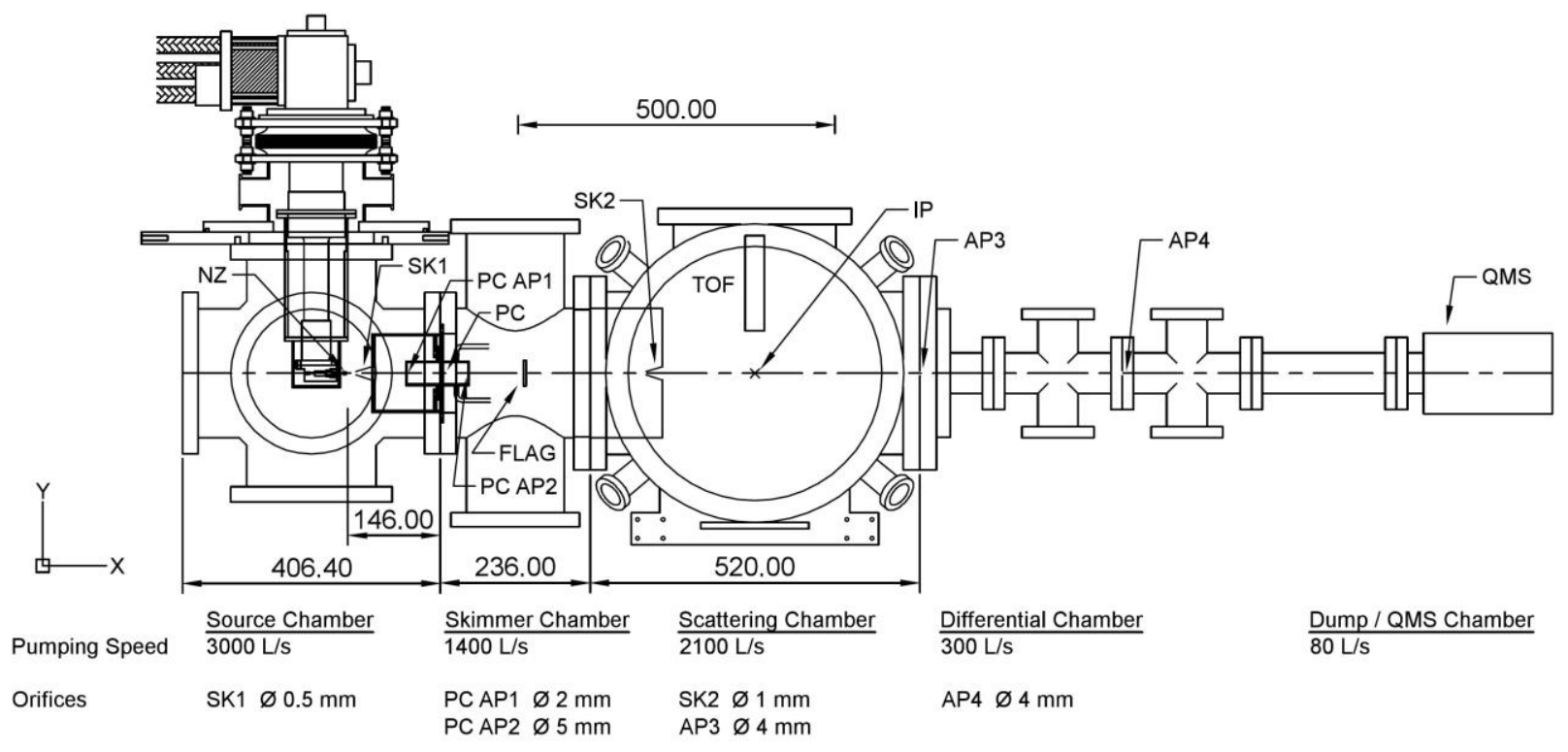

\section{Fig. S1. Schematic of the He droplet apparatus.}

The nominal turbo-molecular pumping speeds in each vacuum chamber are given in $\mathrm{L} / \mathrm{s}$, and the diameters of the skimmers and the apertures are given in $\mathbf{m m}$. Labels used in the figure: $\mathbf{N Z}$ is the cold nozzle; SK1 and SK2 are the skimmers; PC is the pickup chamber with Xe inlet and pressure sensor tubes; PC AP1 and PC AP2 are the pickup cell apertures; IP is the interaction point with the X-ray beam, which enters perpendicular to the plane of the drawing; TOF is the time of flight mass spectrometer; AP3 and AP4 are the downstream apertures; FLAG is the beam shutter; and QMS is the quadrupole mass spectrometer. 


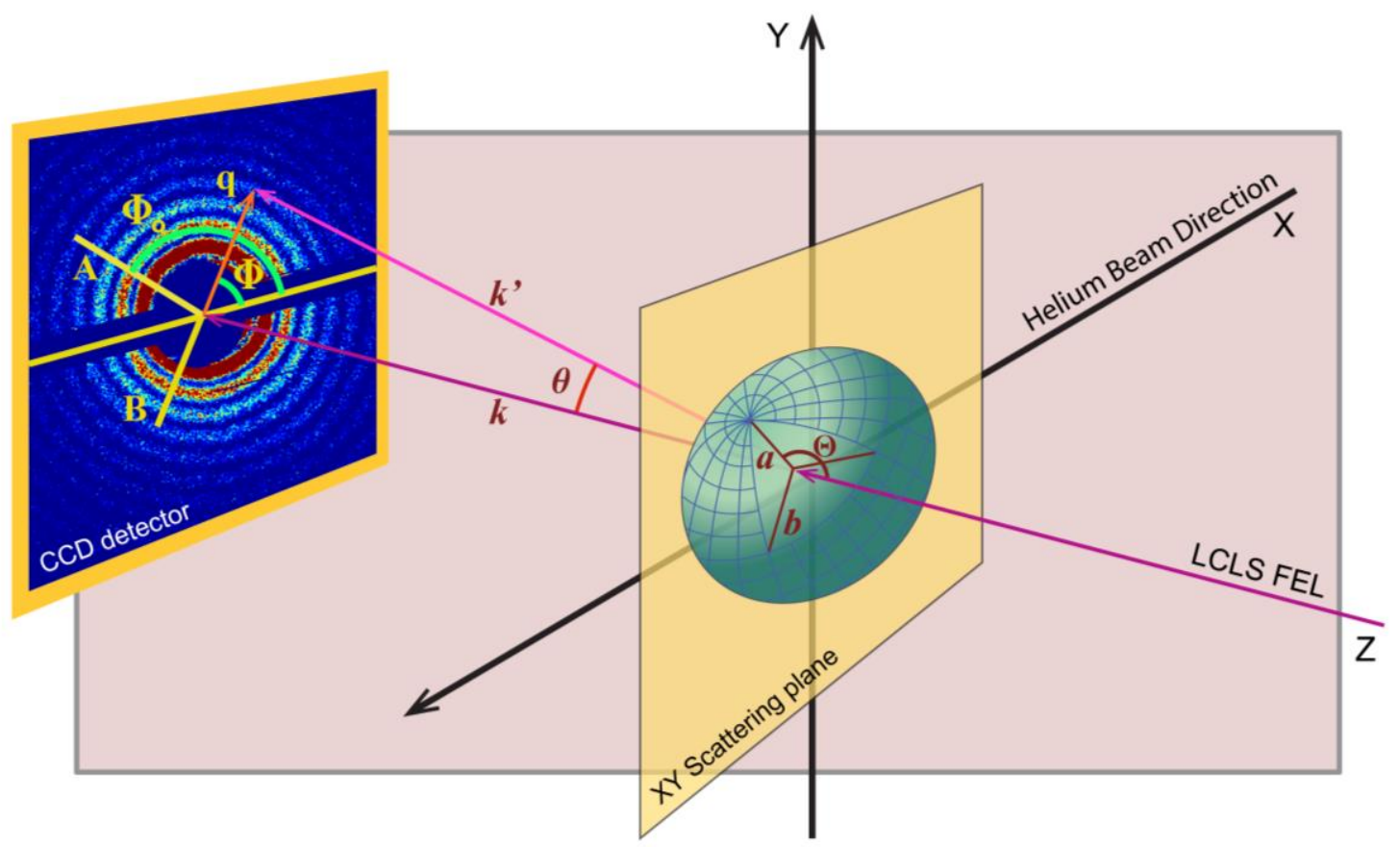

Fig. S2. Scattering of the X-ray beam by a spheroid. 

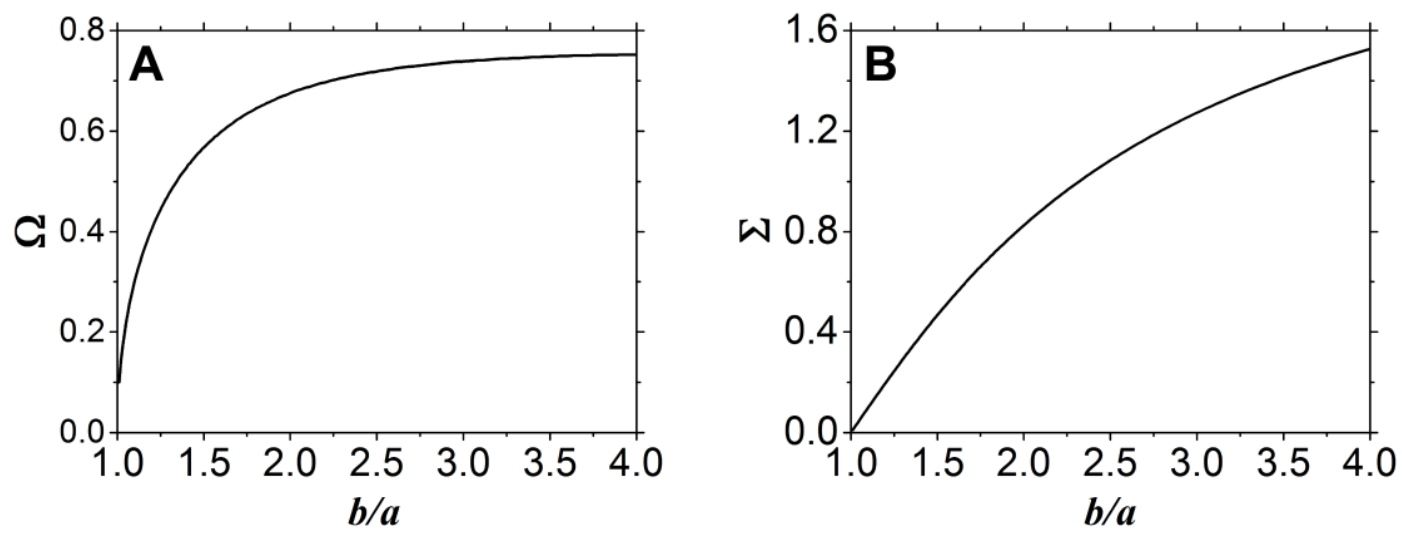

Fig. S3. Aspect-ratio dependence of angular velocity.

(A) Bond number, $\Omega$, and (B) rotation number, $\Sigma$, as functions of the aspect ratio $\boldsymbol{b} / \boldsymbol{a}$ for axially symmetric, rotating helium droplets. 


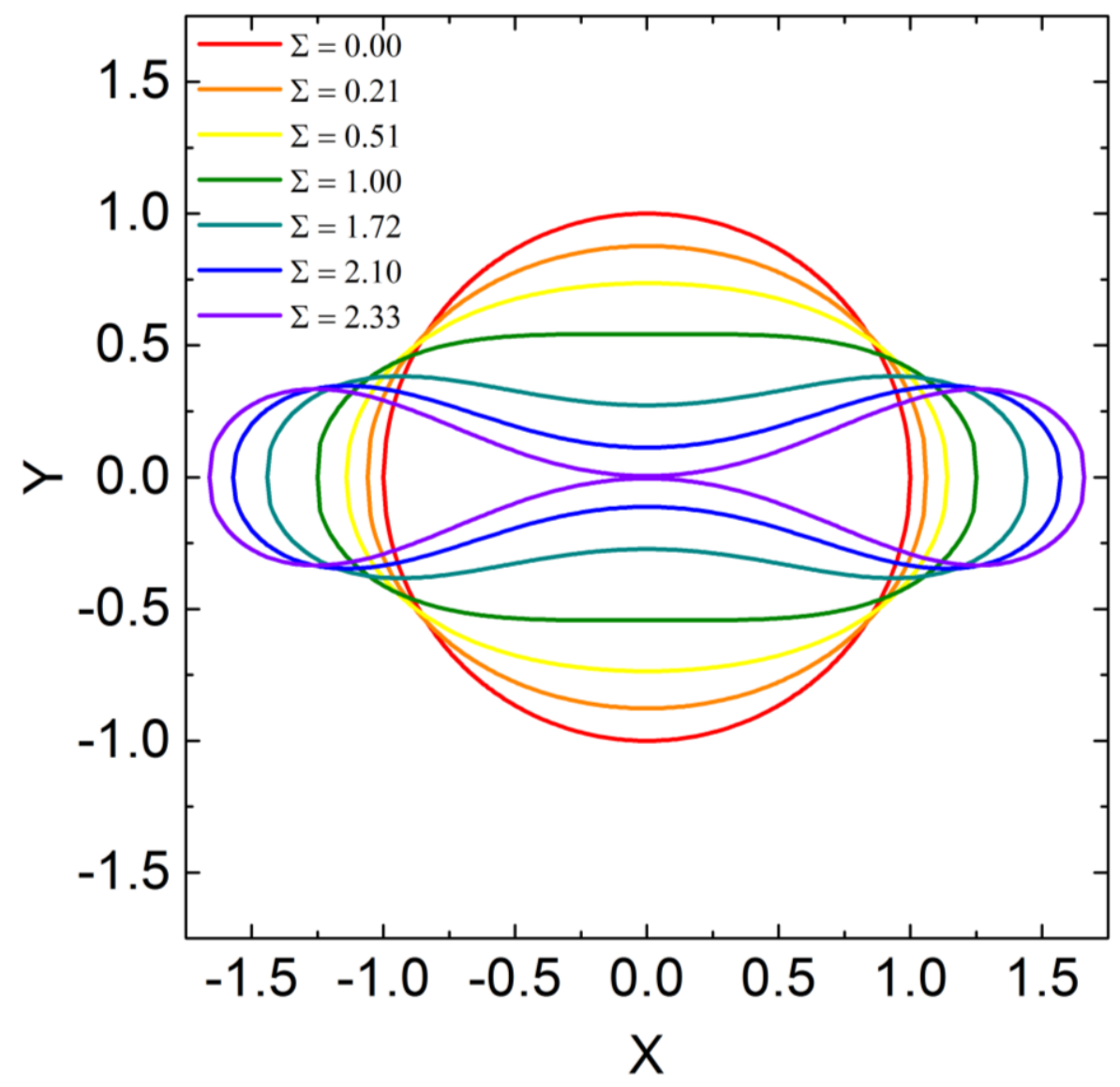

Fig. S4. Cross sections of rotating axisymmetric droplets.

Corresponding values of $\Sigma$ are indicated. All droplets have the same volume. 


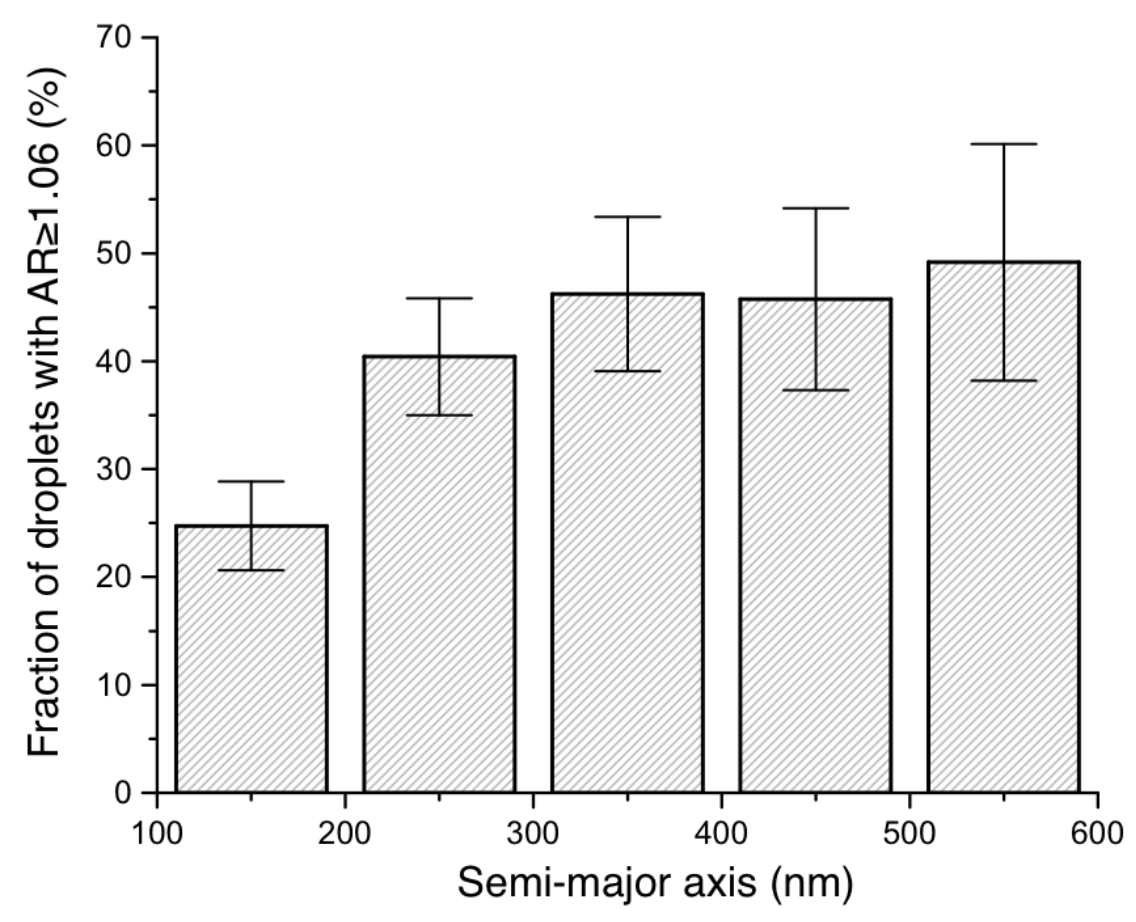

Fig. S5. Size-dependent fraction of non-circular diffraction patterns.

Different droplet sizes are defined by the semi-major axis of the droplet's projection onto the detector. The histogram results from analyzing 663 diffraction images obtained from neat $\mathrm{He}$ droplets. Error bars indicate one-sigma statistical uncertainties. 


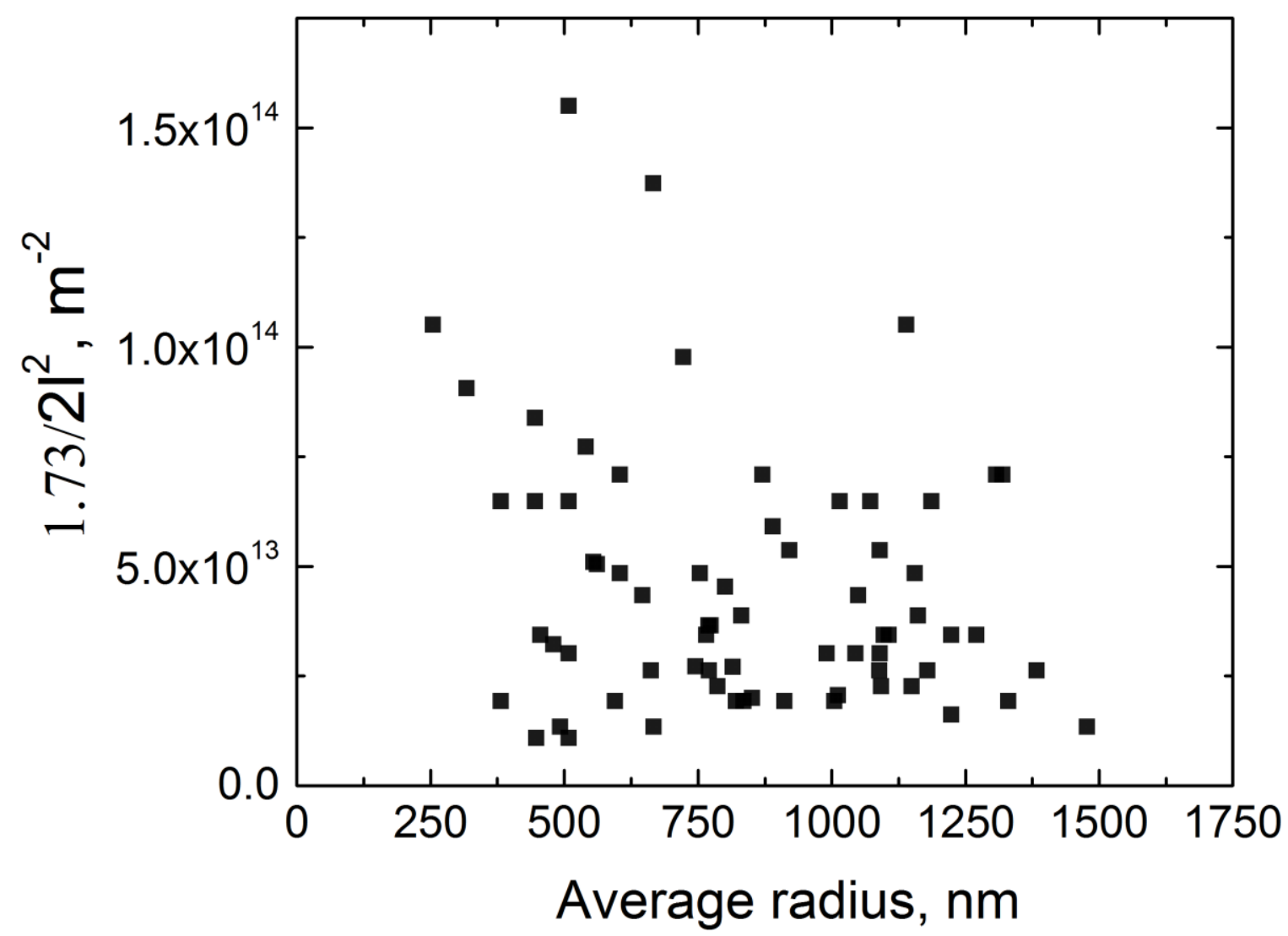

Fig. S6. Vortex Bragg plane density $\sqrt{3} /\left(2 l^{2}\right)$ versus average droplet radius.

The parameter $l$ is the distance between Bragg planes. The plot results from analyzing 69 diffraction images obtained from doped He droplets for which the droplets' radii could be determined. 


\section{References and Notes:}

1. L. Pitaevskii, S. Stringari, Bose-Einstein Condensation, J. Birman et al., Eds. (vol. 116 of The International Series of Monographs on Physics, Clarendon Press, Oxford, 2003).

2. D. R. Tilley, J. Tilley, Superfluidity and Superconductivity (Institute of Physics Publishing, Bristol, UK, 1990).

3. J. F. Allen, A. D. Misener, Flow of liquid helium II. Nature 141, 75 (1938).

4. P. Kapitza, Viscosity of liquid helium below the $\lambda$-point. Nature 141, 74 (1938).

5. L. D. Landau, The theory of superfluidity of helium II. J. Phys. (Moscow) 5, 185-204 (1941).

6. R. P. Feynman, in Progress in Low Temperature Physics, C. J. Gorter, Ed. (North-Holland, Amsterdam, 1955), vol. 1, pp. 1-53.

7. R. J. Donnelly, Quantized Vortices in Helium II, A. M. Goldman, P. V. E. McKlintock, M. Springford, Eds. (vol. 3 of Cambridge Studies in Low Temperature Physics, Cambridge Univ. Press, Cambridge, 1991).

8. S. Grebenev, J. P. Toennies, A. F. Vilesov, Superfluidity within a small helium-4 cluster: The microscopic Andronikashvili experiment. Science 279, 2083-2086 (1998).

9. E. J. Yarmchuk, M. J. V. Gordon, R. E. Packard, Observation of stationary vortex arrays in rotating superfluid helium. Phys. Rev. Lett. 43, 214-217 (1979).

10. G. P. Bewley, D. P. Lathrop, K. R. Sreenivasan, Superfluid helium: Visualization of quantized vortices. Nature 441, 588 (2006).

11. G. H. Bauer, R. J. Donnelly, W. F. Vinen, Vortex configurations in a freely rotating superfluid drop. J. Low Temp. Phys. 98, 47-65 (1995).

12. K. K. Lehmann, R. Schmied, Energetics and possible formation and decay mechanisms of vortices in helium nanodroplets. Phys. Rev. B 68, 224520 (2003).

13. M. Barranco, R. Guardiola, S. Hernández, R. Mayol, J. Navarro, M. Pi, Helium nanodroplets: An overview. J. Low Temp. Phys. 142, 1-81 (2006).

14. M. A. Weilert, D. L. Whitaker, H. J. Maris, G. M. Seidel, Magnetic levitation of liquid helium. J. Low Temp. Phys. 106, 101-131 (1997).

15. L. F. Gomez, E. Loginov, A. F. Vilesov, Traces of vortices in superfluid helium droplets. Phys. Rev. Lett. 108, 155302 (2012).

16. L. Strüder, S. Epp, D. Rolles, R. Hartmann, P. Holl, G. Lutz, H. Soltau, R. Eckart, C. Reich, K. Heinzinger, C. Thamm, A. Rudenko, F. Krasniqi, K.-U. Kühnel, C. Bauer, C.-D. Schröter, R. Moshammer, S. Techert, D. Miessner, M. Porro, O. Hälker, N. Meidinger, N. Kimmel, R. Andritschke, F. Schopper, G. Weidenspointner, A. Ziegler, D. Pietschner, S. Herrmann, U. Pietsch, A. Walenta, W. Leitenberger, C. Bostedt, T. Möller, D. Rupp, M. Adolph, H. Graafsma, H. Hirsemann, K. Gärtner, R. Richter, L. Foucar, R. L. Shoeman, I. Schlichting, J. Ullrich, Large-format, high-speed, x-ray pnCCDs combined with electron and ion imaging spectrometers in a multipurpose chamber for experiments at 4th generation light sources. Nucl. Instrum. Methods A 614, 483-496 (2010).

17. C. Bostedt, J. D. Bozek, P. H. Bucksbaum, R. N. Coffee, J. B. Hastings, Z. Huang, R. W. Lee, S. Schorb, J. N. Corlett, P. Denes, P. Emma, R. W. Falcone, R. W. Schoenlein, G. Doumy, E. P. Kanter, B. Kraessig, S. Southworth, L. Young, L. Fang, M. Hoener, N. Berrah, C. Roedig, L. F. DiMauro, Ultra-fast and ultra-intense x-ray sciences: First results from the Linac Coherent Light Source free-electron laser. J. Phys. B 46, 164003 (2013).

18. T. Gorkhover, M. Adolph, D. Rupp, S. Schorb, S. W. Epp, B. Erk, L. Foucar, R. Hartmann, N. Kimmel, K.-U. Kühnel, D. Rolles, B. Rudek, A. Rudenko, R. Andritschke, A. Aquila, 
J. D. Bozek, N. Coppola, T. Erke, F. Filsinger, H. Gorke, H. Graafsma, L. Gumprecht, G. Hauser, S. Herrmann, H. Hirsemann, A. Hömke, P. Holl, C. Kaiser, F. Krasniqi, J.-H. Meyer, M. Matysek, M. Messerschmidt, D. Miessner, B. Nilsson, D. Pietschner, G. Potdevin, C. Reich, G. Schaller, C. Schmidt, F. Schopper, C. D. Schröter, J. Schulz, H. Soltau, G. Weidenspointner, I. Schlichting, L. Strüder, J. Ullrich, T. Möller, C. Bostedt, Nanoplasma dynamics of single large xenon clusters irradiated with superintense X-ray pulses from the linac coherent light source free-electron laser. Phys. Rev. Lett. 108, 245005 (2012).

19. L. F. Gomez, E. Loginov, R. Sliter, A. F. Vilesov, Sizes of large He droplets. J. Chem. Phys. 135, 154201 (2011).

20. J. P. Toennies, A. F. Vilesov, Superfluid helium droplets: A uniquely cold nanomatrix for molecules and molecular complexes. Angew. Chem. Int. Ed. 43, 2622-2648 (2004).

21. Materials and methods and supporting analysis of the experimental data are available as supplementary materials on Science Online.

22. R. A. Brown, L. E. Scriven, The shape and stability of rotating liquid drops. Proc. R. Soc. London Ser. A 371, 331-357 (1980).

23. S. Chandrasekhar, The stability of a rotating liquid drop. Proc. R. Soc. London Ser. A 286, $1-26(1965)$.

24. R. J. A. Hill, L. Eaves, Nonaxisymmetric shapes of a magnetically levitated and spinning water droplet. Phys. Rev. Lett. 101, 234501 (2008).

25. J. R. Abo-Shaeer, C. Raman, J. M. Vogels, W. Ketterle, Observation of vortex lattices in Bose-Einstein condensates. Science 292, 476-479 (2001).

26. A. L. Fetter, Rotating trapped Bose-Einstein condensates. Rev. Mod. Phys. 81, 647-691 (2009).

27. A. P. Finne, T. Araki, R. Blaauwgeers, V. B. Eltsov, N. B. Kopnin, M. Krusius, L. Skrbek, M. Tsubota, G. E. Volovik, An intrinsic velocity-independent criterion for superfluid turbulence. Nature 424, 1022-1025 (2003).

28. P. M. Walmsley, A. I. Golov, H. E. Hall, A. A. Levchenko, W. F. Vinen, Dissipation of quantum turbulence in the zero temperature limit. Phys. Rev. Lett. 99, 265302 (2007).

29. E. Loginov, L. F. Gomez, A. F. Vilesov, Surface deposition and imaging of large Ag clusters formed in He droplets. J. Phys. Chem. A 115, 7199-7204 (2011).

30. M. Grech, R. Nuter, A. Mikaberidze, P. Di Cintio, L. Gremillet, E. Lefebvre, U. Saalmann, J. M. Rost, S. Skupin, Coulomb explosion of uniformly charged spheroids. Phys. Rev. E 84, 056404 (2011).

31. D. M. Brink, S. Stringari, Density of states and evaporation rate of helium clusters. Z. Phys. D 15, 257-263 (1990).

32. M. Hartmann, R. E. Miller, J. P. Toennies, A. Vilesov, Rotationally resolved spectroscopy of SF6 in liquid helium clusters: a molecular probe of cluster temperature. Phys. Rev. Lett. 75, 1566-1569 (1995).

33. R. J. Donnelly, C. F. Barenghi, The observed properties of liquid helium at the saturated vapor pressure. J. Phys. Chem. Ref. Data 27, 1217-1274 (1998).

34. M. Kerker, The Scattering of Light and Other Electromagnetic Radiation (Academic Press, New York, 1969).

35. T. W. Chen, L. M. Yang, Simple formula for small-angle light scattering by a spheroid. Opt. Commun. 123, 437-442 (1996).

36. H. Lamb, Hydrodynamics (Dover, New York, ed. 6, 1945). 
37. D. L. Whitaker, C. Kim, C. L. Vicente, M. A. Weilert, H. J. Maris, G. M. Seidel, Shape oscillations in levitated HE II drops. J. Low Temp. Phys. 113, 491-499 (1998).

38. C. F. Barenghi, R. J. Donnelly, W. F. Vinen, Thermal excitation of waves on quantized vortices. Phys. Fluids 28, 498-504 (1985).

39. C. F. Barenghi, R. J. Donnelly, W. F. Vinen, Friction on quantized vortices in helium II. A review. J. Low Temp. Phys. 52, 189-247 (1983).

\section{Acknowledgments:}

This work was supported by NSF grant CHE-1112391 (A.F.V.) at USC, by the U.S. Department of Energy, Office of Basic Energy Sciences, (DOE, OBES) Chemical Sciences, Geosciences and Biosciences Division, through Contract No. DE-AC02-05CH11231 and the Max Planck Society by funding the development and operation of the CAMP instrument within the ASG at CFEL. Portions of this research were carried out at the Linac Coherent Light Source, a national user facility operated by Stanford University on behalf of the U.S. DOE, OBES under beam-time grant L549: Imaging of quantum vortices in superfluid helium droplets. S. R. L. and J. P. acknowledge support from the Office of the Secretary of Defense, National Security Science and Engineering Faculty Fellowship. C.B. was partially supported through the PULSE Institute at SLAC National Accelerator Laboratory funded by the U.S. DOE OBES under Contract DEAC02-76SF00515. 